Article

\title{
Boundary Shape Inversion of Two-Dimensional Steady-State Heat Transfer System Based on Finite Volume Method and Decentralized Fuzzy Adaptive PID Control
}

\author{
Liangliang Yang ${ }^{1} \mathbb{D}$, Xiaogang Sun ${ }^{1, *}$ and Yuanli Chu ${ }^{2}$ \\ 1 School of Instrumentation Science and Engineering, Harbin Institute of Technology, Harbin 150001, China; \\ yall21@126.com \\ 2 Harbin Institute of Technology Library, Harbin Institute of Technology, Harbin 150001, China; \\ chuyl@hit.edu.cn \\ * Correspondence: sxg@hit.edu.cn; Tel.: +86-13074589432
}

Received: 26 November 2019; Accepted: 21 December 2019; Published: 23 December 2019

\begin{abstract}
A shape identification scheme was developed to determine the geometric shape of the inaccessible parts of two-dimensional objects using the measured temperatures on their accessible surfaces. The finite volume method was used to calculate the measured point's temperature in the forward problem. In the inversion problem, the decentralized fuzzy adaptive Proportion Integral Differential (PID) control (DFAC) algorithm was used to compensate for the inversion boundary by using the difference between the measurement temperature and the calculation temperature. More accurate inversion results were obtained by introducing the weighted and synthesized normal distribution. In the inversion problem, the effects of the initial guess, the number of measuring points, and the measurement error were studied. The experiment calculation and analysis showed that the methods adopted in this paper still maintain good validity and accuracy with different initial guesses and decrease the number of measuring points and the existence of measurement errors.
\end{abstract}

Keywords: Inverse problem; Inverse Geometry Problem; heat transfer; Fuzzy Adaptive Control PID algorithm; inverse heat conduction problem

\section{Introduction}

In studies of heat conduction problems (forward problems), the geometric shape of the studied object, thermal conductivity, initial conditions, boundary conditions, and inner heat source are all known. The temperature field in the domain can then be directly obtained using numerical heat transfer analysis. Accordingly, if the temperature values at specific points in the system are known but one or more of the initial conditions, boundary conditions, geometry, inner heat source, or thermal conductivity are unknown, these unknowns' details can be estimated using inverse problem methods. There are inverse heat conduction problems (IHCPs) that are well known to be ill posed. The IHCP has been successfully applied in many industrial and engineering fields [1-11].

Inverse geometry problems (IGPs) are considered to be inverse problems in which the geometric shapes of some parts of a studied object are unknown and must be determined based on measured temperature data in other parts of the object. In most cases, these temperatures are measured on the outer surfaces of the solid body using a set of thermocouples or a thermal image scanning system. These IGPs can be distinguished by three important aspects [12]: (1) defects in detection [13,14], (2) shape design [15-17], and (3) identification of boundaries [18]. In this paper, we focus on the boundary identification problem. 
IGPs, as one branch of IHCPs, have been studied by a significant number of scholars with different optimized algorithms. The optimized algorithms, which are used to minimize the objective function, are mainly divided into gradient- and non-gradient-based optimization algorithms. Gradient-based optimization algorithms include the conjugate gradient method (CGM) $[6,17,19,20]$, the Levenberg-Marquardt method (L-MM), the steepest descent method (SDM), and the Gauss-Newton algorithm. Non-gradient-based optimization algorithms include genetic algorithms (GAs), neural network algorithms (NNAs), particle swarm optimization (PSO), the artificial bee colony algorithm (ABCA), and so forth.

Huang et al. [19-21] used the BEM with CGM/LM to study irregular geometrical inverse problems. The boundary identification problems they studied included frost growth on an evaporating tube and irregular geometric boundary identification with time in two-dimensional unsteady heat conduction problems. Fan et al. [18] determined the geometric shape of flat plate using the CGM. Fan et al. [22] used the FEM and the CGM to study the internal defects of a two-dimensional pipeline and discussed the effects of the initial guess and measurement errors on the inversion results. Li Bin et al. [23,24] used the BEM and the CGM to study the geometrical shapes of the inner walls of a two-dimensional cylinder. They discussed the influence on the inversion results of the inner wall shape of the initial guess, the measurement error, and the number of measured points. Tian [25] solved a two-dimensional steady-state thermal boundary problem using PSO. Xiao, Lin et al. $[26,27]$ introduced an improved PSO algorithm that can search for more solutions simultaneously, which makes it possible to give an unbiased estimate that provides a better way to find the optimal solution in the search space. Wang et al. [28] applied a hybrid CGM with SPSO to solve two-dimensional steady-state boundary shape identification, which improved the convergence speed. Partridge et al. [29] applied the GA to estimate the size and location of a dermatome based on the information of skin surface temperature. Zhu [30] applied the CGM and GA to study the inversion of two-dimensional boundary conditions and analyzed the shortcomings of the CGM and GA.

The CGM is currently the most popular gradient-based optimization algorithm. However, the CGM, which is a local search algorithm, also has drawbacks, such as that it easily converges to a locally optimal solution. The inversion result depends crucially on the initial guess. More importantly, when the temperature measurement information is not complete or there is a large measurement error, the inversion results obtained based on the gradient optimization method may deteriorate. In addition, when the number of inversion points is larger, the calculation of the gradient matrix is difficult and time consuming, which directly affects the engineering application of gradient-based optimization algorithms.

Non-gradient-based optimization algorithms are usually global search algorithms and have good adaptability to overcome the difficulty of gradient-based optimization algorithms easily converging to a local minimum. Moreover, they do not involve the calculation of a Jacobian partial derivative matrix in the inversion process. However, this class of methods often has high computational costs in the search process and has a slow convergence rate in the latter stage, which limits its application in IHCPs. In particular, when the measurement information is incomplete or there is a large measurement error, the inversion results obtained according to such methods will also have some gaps.

Decentralized fuzzy inference was successfully applied to invert thermal conductivity by Wang et al. [2], and the accuracy and stability of the algorithm were verified. There are few reports on the application of the decentralized fuzzy adaptive PID control (DFAC) to IGPs. In this study, the finite volume method (FVM) was used to solve the forward problem, the DFAC algorithm was used to compensate for the initial guess of the estimate boundary in order to minimize the residual between the calculated and the measured values of the temperature, and the true geometry boundary was obtained. To study the effectiveness and stability of the proposed method, the effects of initial guesses, measurement points, and measurement errors on the results were analyzed and discussed. 


\section{Forward Problem Description (Mathematical Formulation)}

The two-dimensional steady-state heat transfer system (domain $\Omega$ ) is shown in Figure 1, which was based on heat transfer from the walls of industrial equipment in real operational environments. The shape of the accessible boundary $\Gamma$ was given but the inaccessible inner boundary $\Gamma_{I}$ was unknown.

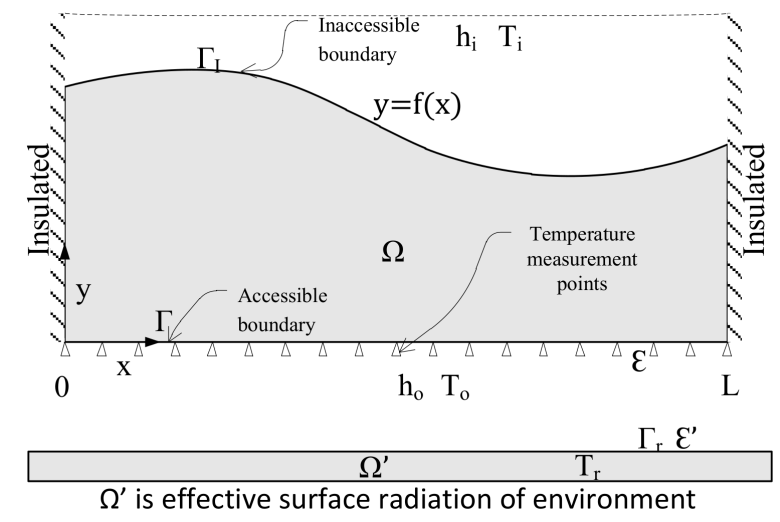

Figure 1. Physical model of inner wall boundary identification of thermal equipment.

The boundary conditions at $x=0$ and $x=L$ are adiabatic; the boundary condition of the unknown boundary $\Gamma_{I}(y=f(x))$ is the robin boundary condition with a fluid temperature of $T_{I}$ and a heat transfer coefficient of $h_{i}$. The boundary condition of $\Gamma(y=0)$ is a combination of convection and radiation, where the ambient temperature is $T_{0}$, the heat transfer coefficient is $T_{0}$, and the absorptivity is $\varepsilon$. The points marked " $\Delta$ " are temperature sensor positions, assuming a uniform distribution of $M$ measurement points set on $\Gamma$. For the domain $\Omega^{\prime}$, which is the effective absorptivity model of the physical environment (surface radiation), the boundary condition at $\Gamma_{r}$ is constant temperature $T_{r}$ and the effective absorptivity is $\varepsilon^{\prime}$.

Assuming that the material properties of the problem domain $\Omega$ are a constant thermal conductivity $k, \Omega$ and $\Omega^{\prime}$ are gray bodies, and $\varepsilon=\varepsilon^{\prime}$, the governing equation of this two-dimensional steady-state heat conduction system can be expressed as

$$
\frac{\partial^{2} T}{\partial x^{2}}+\frac{\partial^{2} T}{\partial y^{2}}=0 \text { in } \Omega
$$

The boundary conditions are as follows:

When $x=0$ and $x=L$, the heat conduction equation is

$$
\left.\frac{\partial T}{\partial n}\right|_{x=0, x=L}=0 .
$$

When $y=0$, the heat conduction equation is

$$
-\left.k \frac{\partial T}{\partial n}\right|_{y=0}=h_{o}\left(T_{o}-T_{x i}\right)+\varepsilon \sigma^{\prime}\left(T_{r}^{4}-T_{x i}^{4}\right)
$$

Namely,

$$
-\left.k \frac{\partial T}{\partial n}\right|_{y=0}=h_{o}\left(T_{o}-T_{x i}\right)+h_{r}\left(T_{r}-T_{x i}\right), i=0, \ldots, M x i \in \Gamma
$$

where the equivalent radiative heat transfer coefficient $h_{r}=\varepsilon \sigma^{\prime}\left(T_{r}{ }^{2}+T_{x i}{ }^{2}\right) * T_{r}+T_{x i}$. The Stefan-Boltzmann constant $\sigma^{\prime}$ is $5.669 \times 10^{-8} \mathrm{~W} /\left(\mathrm{m}^{2} \mathrm{k}^{4}\right)$. 
When $y=f(x)$, the heat conduction equation is

$$
-\left.k \frac{\partial T}{\partial n}\right|_{y=f(x)}=h_{i}\left(T-T_{I}\right), x \in \Gamma_{I}
$$

The FVM was used to solve the forward problem of Equations (1) and (2). To simplify the forward problem and remeshing process for IGPs, the mesh grid of FVM was fixed to a small value such as $0.001 \mathrm{~m}$.

\section{The Inverse Problem}

\subsection{Objective Function of Inverse Problem}

For the physical model of Figure 1, the inaccessible boundary $\Gamma_{I}$ is unknown, while everything else in the forward problem is given. We assumed that $M$ measurement points are evenly distributed on the boundary $\Gamma$. There are

$$
T\left(x_{i}, 0\right)=T_{i}, i=1,2, \ldots, M \text {. }
$$

Then, the geometry identification problem can be transformed to an extreme value problem, and the objective function can be expressed as

$$
J=\sum_{i=1}^{M}\left[T_{i}-T_{i}^{*}\right]^{2}
$$

where $T_{i}^{*}$ is the measured temperature along the $\Gamma$, and each $T_{i}$ is the calculated value of the forward problem based on the inversion boundary of the unknown boundary. The solutions of IGPs were obtained by minimizing the objective function (7) using the DFAC.

\subsection{Stop Criterion}

The stopping criterion of the iteration was selected as follows. If the problem contains no measurement errors, the inverse iterative process will terminate when this inequality is satisfied:

$$
J=\sum_{i=1}^{M}\left[T_{i}-T_{i}^{*}\right]^{2}<\varepsilon_{o b j}
$$

where $\varepsilon_{o b j}$ is a small positive number to be specified as a solution parameter, such as 0.001 .

The known boundary temperatures are measured and always contain measurement errors in practical engineering applications. Therefore, we did not expect the objective function (Equation (12)) to be equal to zero at the final iteration. We assumed that the measurement temperature was composed of the exact temperature and random error, as

$$
T_{i}^{*}=T_{i}^{e x}+\omega \sigma, i=1,2, \ldots, M
$$

where $\sigma$ is the maximum error of the measured temperature, and $\omega$ is a normal distribution random variable with zero mean and unit standard variation.

\subsection{Decentralized Fuzzy Adaptive PID Control Method}

\subsubsection{Decentralized Fuzzy Adaptive PID Control Inversion System}

The decentralized fuzzy adaptive PID control inversion system for IGPs was established as shown Figure 2. Then, a multiple-input multiple-output decentralized fuzzy adaptive PID control system was established. The system included $M$ one-dimensional fuzzy adaptive PID control units (FACUs). $\Delta T_{i}$, 
which is difference between the calculated temperature and the measured temperature, was used to correspondingly compensate for the guess of the boundary. $\Delta T_{i}$ is

$$
\Delta T_{i}=T_{i}-T_{i}^{*}, i=1,2, \ldots, M
$$

where $T_{i}{ }^{*}$ is the measured temperature at the $i$ th measurement point, and $T_{i}$ is the calculated temperature by solving the forward problem.

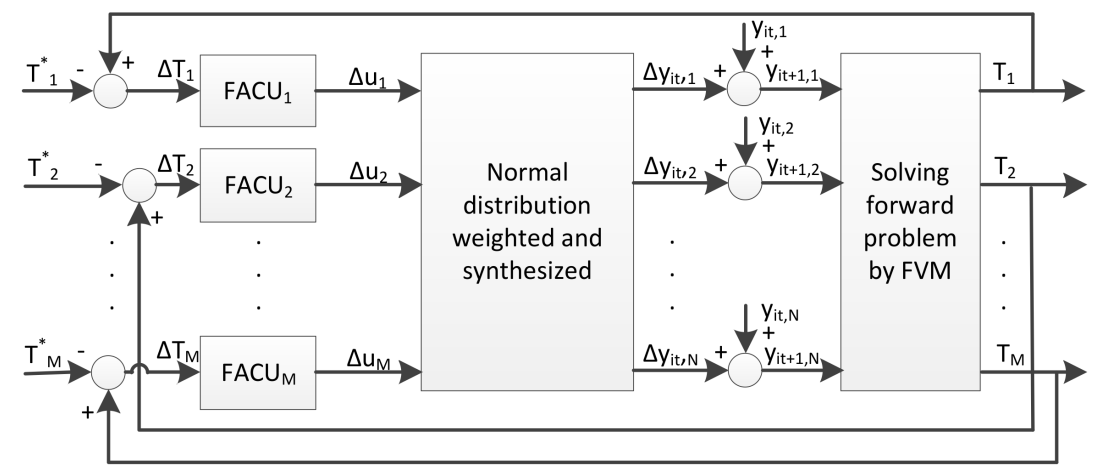

Figure 2. Decentralized fuzzy adaptive Proportion Integral Differential (PID) control inversion system.

$\Delta T_{i}$ is the input value of $F A C U_{i}$, and the components $\Delta u_{i}$ are obtained. Then, after weighting and synthesizing, the compensations of $\Delta y_{i t, j}$ are generated, and the new inverse boundary $y_{i t+1, j}$ is updated as follows:

$$
y_{i t+1, j}=y_{i t, j}+\Delta y_{i t, j}, j=1,2, \ldots, N
$$

where it denotes the iteration number, and $N$ represents the unknown boundary discrete points.

\subsubsection{Fuzzy Adaptive Control PID Units}

Each independent measurement point is a single FACU. The FACU model is shown in Figure 3.

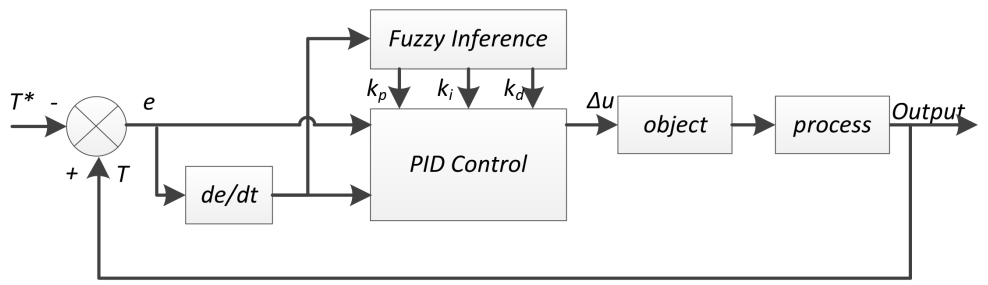

Figure 3. Fuzzy adaptive PID control system model.

The measurement and inverted information in the heat conduction system have certain spatial distribution characteristics. Therefore, the inverse heat conduction problem can also be regarded as a kind of feedback control problem, that is, a closed-loop system, where the observation results (measured temperature) of the system are the setting value, the parameters to be solved are the controlled object, and the calculated temperature of the positive problem is the sensor measurement information. $e=\Delta T$ is obtained according to Equation (10); if $\Delta T>0$, in order to eliminate or reduce the deviation, it is necessary to increase $y$; if $\Delta T<0$, to eliminate or reduce the deviation, it is necessary to decrease $y$. Then, $\Delta u$ is defined as follows:

$$
\Delta u=k_{p} \Delta T+k_{i} \Delta \sum_{h=0}^{i t} \Delta T
$$


where $k_{p}$ and $k_{i}$ are the feedback factor, and $k_{p}$ and $k_{i}$ are adaptive updates by fuzzy inference.

\subsubsection{Weighting and Synthesizing Scheme}

$\Delta u_{i}$ is the compensation for the boundary of all nodes at an unknown boundary when only the $i$ th measured temperature is considered. Actually, the value of all nodes at the unknown boundary has a different effect on the temperature of all measured points. So, the whole measured temperature should be considered when compensating for the unknown boundary implementation. The weighting and synthesizing scheme are performed by Equation (13):

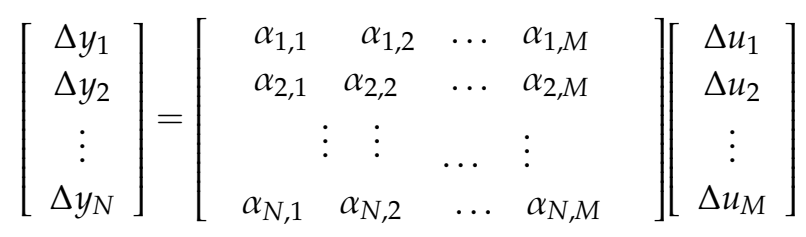

where $\alpha_{j, i}$ is the weighting factor, which reflects the influence of $j$ th discrete point of $f(x)$ on the $i$ th temperature at the measured point; the larger $\alpha_{j, i}$ is, the greater the influence. Based on the normal distribution weighting method, the weighting coefficient $\alpha_{j, i}$ is determined by Equation (14):

$$
\alpha_{j, i}=\frac{\exp \left[-\left(\frac{\left|x_{j}-x_{i}\right|}{\varnothing}\right)^{2}\right]}{\sum_{i}^{M} \exp \left[-\left(\frac{\left|x_{j}-x_{i}\right|}{\varnothing}\right)^{2}\right]}, j=1,2, \ldots, N
$$

where $x_{i}, x_{j}$ are the $x$-axis values of the inversion and measurement points, and $\Phi$ is the variance coefficient of normal distribution. The larger $\Phi$ is, the smoother the distribution curve of the weighting coefficient is, the more measurement information is effectively used in the inversion process, but the slower the inversion speed is; the smaller $\Phi$ is, the more centralized the distribution of the weighting coefficient is, the less measurement information is effectively used, but the faster the inversion speed is.

\subsection{Implementation of Inverse Geometry Problem}

The process for solving the IGPs is as follows:

(1) Initialize the guess of the unknown boundary and set the iterative time it $=0$.

(2) Solve the forward problem by FVM: solve Equations (1) and (2) using the FVM and get the calculated temperature $T_{i}$ at the inspection surface.

(3) Calculate the objective function Equation (7) and judge if the specified stopping criterion is satisfied (Equation (8)). If not, go to step 4.

(4) Calculate the optimal positions $y_{i}{ }^{n+1}$ of the unknown boundary according to the temperature measurement points' coordinates using Equation (11).

(5) Set it $=i t+1$ and repeat steps $2-5$ until the stop criterion is satisfied.

\section{Experiment and Analysis}

In the experiment, a two-dimensional flat plate furnace wall was considered, as shown in Figure 1. The boundary conditions were given except that the inaccessible boundary was unknown. $T_{o}=20^{\circ} \mathrm{C}$, $h_{o}=10 \mathrm{~W} /\left(\mathrm{m}^{2} \mathrm{~K}\right), T_{i}=150{ }^{\circ} \mathrm{C}, h_{i}=1000 \mathrm{~W} /\left(\mathrm{m}^{2} \mathrm{~K}\right)$, and $\varepsilon=\varepsilon^{\prime}=0.8$. The thermal conductivity $k=5 \mathrm{~W} /(\mathrm{m} \cdot \mathrm{K}), c=500 \mathrm{KJ} /(\mathrm{Kg} \cdot \mathrm{K})$, and $\rho=7930 \mathrm{Kg} /\left(\mathrm{m}^{3}\right)$. The measurement points $M$ were located evenly spaced along the accessible boundary.

The function of the unknown boundary was defined as follows:

Case 1:

$$
y=f(x)=0.1+\frac{2}{9} x, x \in[0,0.9]
$$


Case 2:

$$
y=f(x)=0.2-0.1 * \sin \left(\frac{2 \pi x}{0.9}\right), x \in[0,0.9]
$$

\subsection{Impacts of Initial Guess}

The measurement error $\sigma=0.0{ }^{\circ} \mathrm{C}$, and the measurement point was taken as $M=41$. The initial guesses were set as $0.1,0.2$, and 0.3 for cases 1 and 2 . The inversion results of the different initial guesses are shown in Figures 4 and 5, and the average relative error is shown in Table 1.

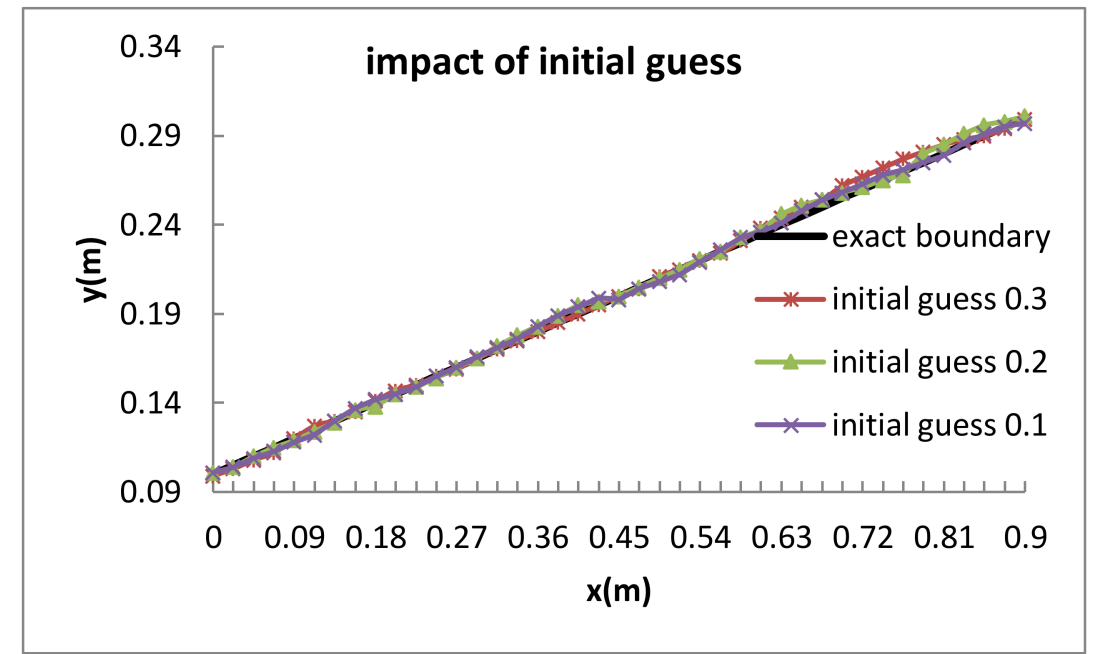

Figure 4. Identification results of different initial guess boundaries of case 1 .

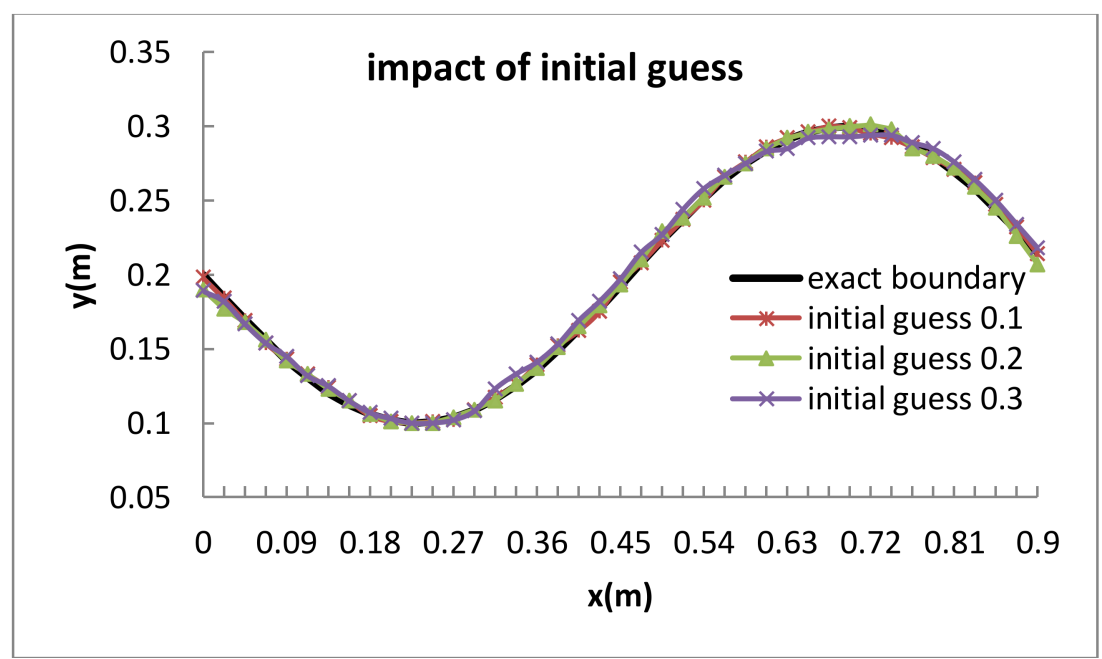

Figure 5. Identification results of different initial guess boundaries of case 2 .

Table 1. Impacts of initial guess of cases 1 and 2.

\begin{tabular}{ccc}
\hline Initial Guess (m) & Case 1 Average Relative Error (\%) & Case 2 Average Relative Error (\%) \\
\hline 0.1 & 0.92 & 0.95 \\
0.2 & 0.95 & 1.04 \\
0.3 & 0.89 & 1.63 \\
\hline
\end{tabular}

Table 1 and Figures 4 and 5 show that the identification results were insensitive to the initial guesses of the unknown boundaries. 


\subsection{Impact of the Number of Measurement Points}

The measurement error was $\sigma=0.1^{\circ} \mathrm{C}$, and the measurement points were taken as $M=11,21$, and 41. The inversion results of different numbers of measurement points are shown in Figures 6 and 7 , and the average relative error is shown in Table 2.

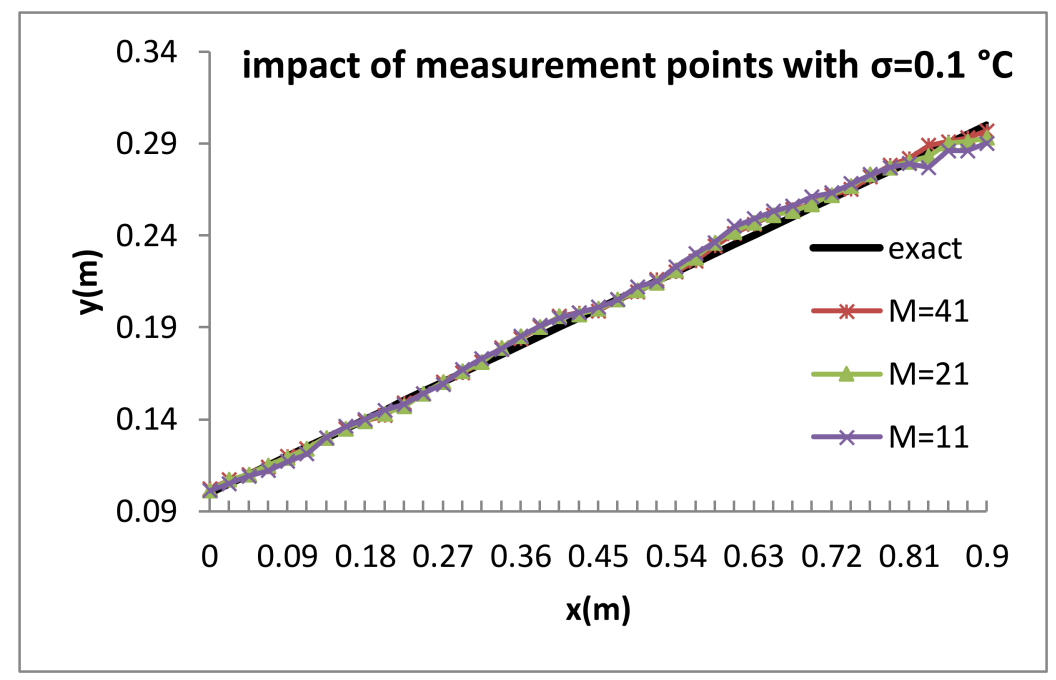

Figure 6. Identification results of different measurement points for inversion results of case 1.

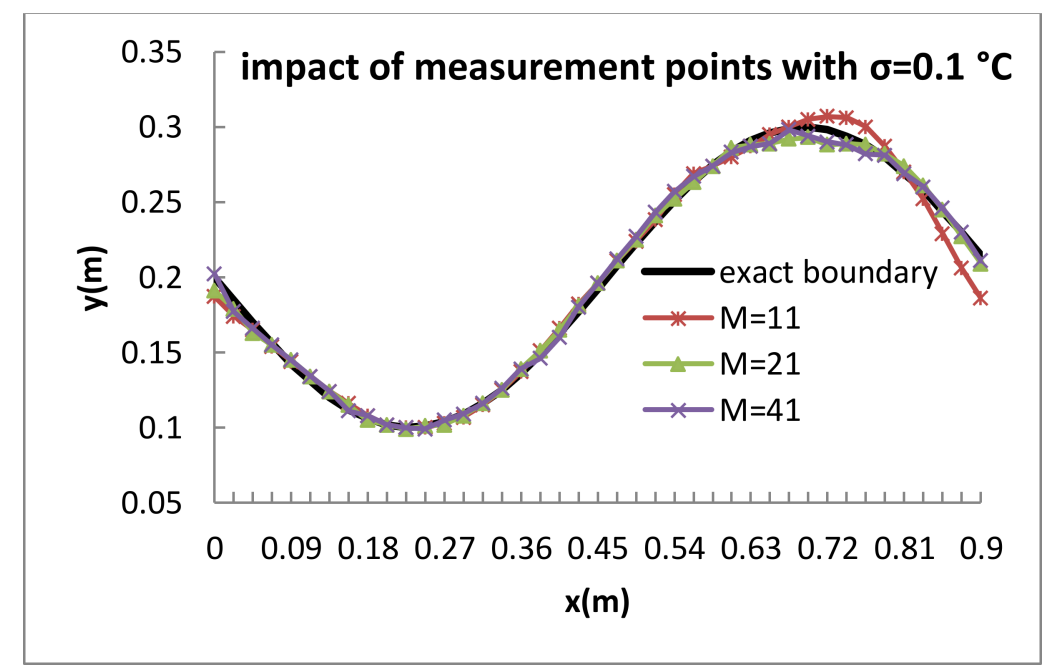

Figure 7. Identification results of different measurement points for inversion results of case 2.

Table 2. Impacts of different measurement points of cases 1 and 2.

\begin{tabular}{ccc}
\hline Measurement Points & Case 1 Average Relative Error (\%) & Case 2 Average Relative Error (\%) \\
\hline 11 & 1.6 & 2.5 \\
21 & 1.1 & 1.6 \\
41 & 1.0 & 1.4 \\
\hline
\end{tabular}

The results are as follows:

Case 1: When $M=11$, the average relative error was $1.6 \%$; when $M=21$, the average relative error was $1.1 \%$; and when $M=41$, the average relative error was $1.0 \%$.

Case 2: When $M=11$, the average relative error was $2.5 \%$; when $M=21$, the average relative error was $1.6 \%$; and when $M=41$, the average relative error was $1.4 \%$. 
By increasing the measuring points, the average relative error decreased and the inversion accuracy improved.

\subsection{Impact of Measurement Error}

The measurement point was set as $M=41$, and the initial guess $=0.1 \mathrm{~m}$. Impacts of different measurement errors $\left(\sigma=0.0,0.1,0.5,1\right.$, and $2{ }^{\circ} \mathrm{C}$ for case 1 and $\sigma=0.0,0.1,0.5$, and $1{ }^{\circ} \mathrm{C}$ for case 2$)$ were analyzed. The inversion results of different measurement errors are shown in Figures 8 and 9.

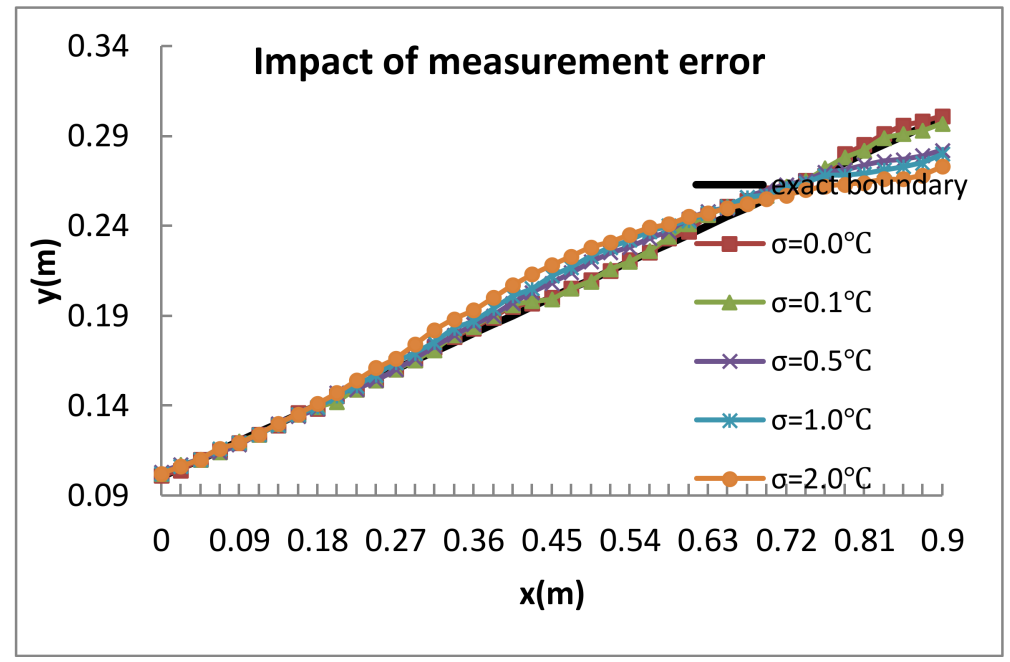

Figure 8. Identification results of different measurement errors for inversion results of case 1.

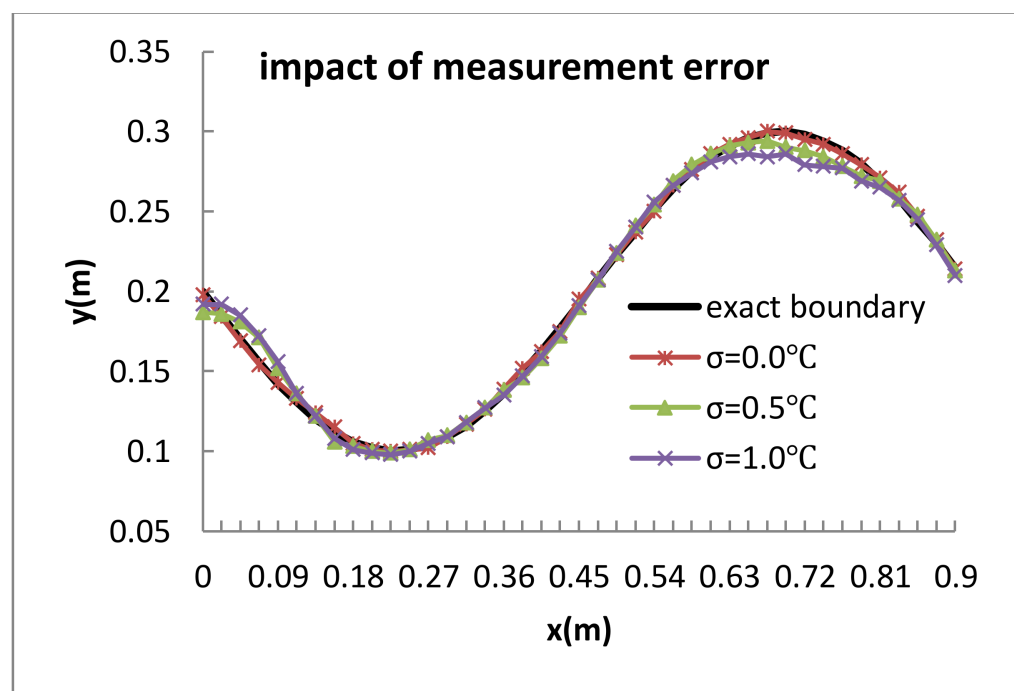

Figure 9. Identification results of different measurement errors for inversion results of case 2.

The results are as follows:

Case 1:

- when $\sigma=0.0^{\circ} \mathrm{C}$, the average relative error was $0.92 \%$;

- when $\sigma=0.1^{\circ} \mathrm{C}$, the average relative error was $1.03 \%$;

- when $\sigma=0.5^{\circ} \mathrm{C}$, the average relative error was $2.35 \%$;

- when $\sigma=1.0^{\circ} \mathrm{C}$, the average relative error was $3.03 \%$;

- when $\sigma=2.0^{\circ} \mathrm{C}$, the average relative error was $4.40 \%$. 
Case 2:

- when $\sigma=0.0^{\circ} \mathrm{C}$, the average relative error was $0.95 \%$;

- when $\sigma=0.5^{\circ} \mathrm{C}$, the average relative error was $2.31 \%$;

- when $\sigma=1.0^{\circ} \mathrm{C}$, the average relative error was $2.79 \%$.

The results showed that when there were some measurement errors, the inversion results were still satisfactory. When the measurement error was less than $1^{\circ} \mathrm{C}$, the accuracy of the inversion solution could still meet the general engineering requirements, and the larger the measurement error, the more distorted the inversion results. Therefore, it was beneficial to improve the inversion results to improve the measurement accuracy as much as possible in the experiment.

\section{Conclusions}

In this study, the decentralized fuzzy adaptive PID control algorithm and finite volume method were used for inversion of the unknown boundary in a two-dimensional steady-state heat transfer system. Several effect factors were considered, involving different initial guess boundaries, measurement points, and measurement errors. The results of the experiment showed that identification results can be satisfactory even if there are measurement errors. Also, the identification results are insensitive to the initial guesses of the unknown boundaries and the number of measurement points. Through the calculation and analysis of the experiment, the stability and accuracy of the boundary inversion algorithm were verified.

Author Contributions: Conceptualization and writing-original draft preparation, L.Y.; investigation, L.Y.; writing-review and editing, L.Y.; methodology, L.Y.; retrieval and proofreading, Y.C.; supervision and funding acquisition, X.S. All authors have read and agreed to the published version of the manuscript.

Funding: This research was funded by the National Natural Science Foundation of China (61875046) and the National Key Foundation for Exploring Scientific Instrument of China (2013YQ470767).

Acknowledgments: The authors would like to thank Jingxin Cui for the helpful discussion, as well as Jingxin Cui and Pengyu Chen for performing the experiments.

Conflicts of Interest: The authors declare no conflict of interest.

\section{References}

1. Lotfi, M.; Mezrigui, L.; Heyd, R. Study of heat conduction through a self-heated composite cylinder by Laplace transfer functions. Appl. Math. Model. 2016, 40, 10360-10376. [CrossRef]

2. Wang, S.; Zhang, L.; Sun, X.; Jia, H. Inversion of thermal conductivity in two-dimensional unsteady-state heat transfer system based on boundary element method and decentralized fuzzy inference. Complexity 2018, 2018, 8783946. [CrossRef]

3. Wang, S.; Zhang, L.; Sun, X.; Jia, H. Solution to Two-Dimensional Steady Inverse Heat Transfer Problems with Interior Heat Source Based on the Conjugate Gradient Method. Math. Probl. Eng. 2017, 2017, 286134. [CrossRef]

4. Hetmaniok, E. Inverse problem for the solidification of binary alloy in the casting mould solved by using the bee optimization algorithm. Heat Mass Transf. 2016, 52, 1369-1379. [CrossRef]

5. Feischl, M.; Gantner, G.; Haberl, A.; Praetorius, D.; Führer, T. Adaptive boundary element methods for optimal convergence of point errors. Numer. Math. 2016, 132, 541-567. [CrossRef]

6. Cui, M.; Duan, W.; Gao, X. Conjugate Gradient Method Based on Complex-variable-differentiation Method and Its Application for Identification of Boundary Conditions in Inverse Heat Conduction Problem. CIESC J. 2015, S1, 106-110.

7. Sriram, S.B.; Sravan, S.; Gnanasekaran, N. Numerical Estimation of Heat Flux and Convective Heat Transfer Coefficient in a One Dimensional Rectangular Plate by Levenberg-Marquardt Method. Indian J. Sci. Technol. 2016, 9. [CrossRef]

8. Kosaka, M.; Monde, M. Simultaneous measurement of thermal diffusivity and thermal conductivity by means of inverse solution for one-dimensional heat conduction (anisotropic thermal properties of CFRP for FCEV). Int. J. Thermophys. 2015, 36, 2590-2598. [CrossRef] 
9. Mohebbi, F.; Sellier, M.; Rabczuk, T. Estimation of linearly temperature-dependent thermal conductivity using an inverse analysis. Int. J. Therm. Sci. 2017, 117, 68-76. [CrossRef]

10. Huang, S.J. An Ant Colony Optimization Algorithm Suitable for Searching Heat Source Location in IHCP. J. Eng. Thermophys. 2013, 34, 694-697.

11. Yang, L.; Sun, B.; Sun, X. Inversion of Thermal Conductivity in Two-Dimensional Unsteady-State Heat Transfer System Based on Finite Difference Method and Artificial Bee Colony. Appl. Sci. 2019, 9, 4824. [CrossRef]

12. Hożejowski, L. Trefftz method for an inverse geometry problem in steady-state heat conduction. J. Appl. Math. Comput. Mech. 2016, 15, 41-52. [CrossRef]

13. Mahmud, K.; Mohsen, D.A. Inclusion Identification by Inverse Application of Boundary Element Method, Genetic Algorithm and Conjugate Gradient Method. Am. J. Appl. Sci. 2008, 5, 1158-1166.

14. Fazeli, H.; Mirzaei, M. Shape identification problems on detecting of defects in a solid body using inverse heat conduction approach. J. Mech. Sci. Technol. (FEM CGM) 2012, 26, 3681-3690. [CrossRef]

15. Lan, C.H.; Cheng, C.H.; Wu, C.Y. Shape Design for heat conduction problems using curvilinear grid generation, conjugate gradient, and redistriution methods. Numer. Heat Transf. Part A 2001, 39, 487-510. [CrossRef]

16. Morimoto, K.; Kinoshita, H.; Suzuki, Y. Adjoint-based shape optimization of fin geometry for heat transfer enhancement in solidification problem. J. Therm. Sci. Technol. 2016, 11, JTST0040. [CrossRef]

17. Huang, C.H.; Chiang, C.C.; Chou, S.K. Inverse geometry design problem in optimizing hull surfaces. J. Ship Res. 1998, 42, 79-85.

18. Fan, C.; Zhang, M.; Hu, S.; Yang, L.; Sun, F. Identification of plate surface geometry a numerical and experimental study. Numer. Heat Transf. Part B 2012, 61, 52-70. [CrossRef]

19. Huang, C.H.; Chao, B.H. An inverse geometry problem in identifying irregular boundary configurations. Int. J. Heat Mass Transf. 2009, 40, 2045-2053. [CrossRef]

20. Huang, C.H.; Chaing, M.T. A three-dimensional inverse geometry problem in identifying irregular boundary configurations. Int. J. Therm. Sci. 2009, 48, 502-513. [CrossRef]

21. Huang, C.H. An Inverse Geometry Problem in Estimating Frost Growth on an Evaporating Tube. Heat Mass Transf. 2002, 38, 615-623. [CrossRef]

22. Fan, C.; Sun, F.; Yang, L. An algorithm study on the identification of a pipeline's irregular inner boundary based on thermographic temperature measurement. Meas. Sci. Technol. 2009, 18, 2170-2177. [CrossRef]

23. Li, B.; Liu, L.H. A Geometric Boundary Identification Algorithm for Thermal Problem Based on Boundary Element Discretization. J. China Electromechan. Eng. 2008, 20, 38-43.

24. Li, B. Boundary Element Method for Geometric Inverse Problem of Thermal Conductivity. Master's Thesis, Harbin Institute of Technology, Harbin, China, 2008.

25. Tian, N. Research and Application of Numerical Solution of Inverse Problem for Partial Differential Equation. Ph.D. Thesis, Jiangnan University, Wuxi, China, 2012.

26. Xiao, H.; Li, P. Improved Quantum Behavior Particle Swarm Optimization Algorithm and Its Application. Inf. Control 2016, 45, 157-164.

27. Lin, W. Research on Improved Particle Swarm Optimization Algorithm and Its Application. Ph.D. Thesis, East China University of Science and Technology, Shanghai, China, 2014.

28. Wang, S.; Jia, H.; Sun, X.; Zhang, L. Two-Dimensional Steady-State Boundary Shape Inversion of CGM-SPSO Algorithm on Temperature Information. Adv. Mater. Sci. Eng. 2017, 2017, 2461498. [CrossRef]

29. Partridge, P.W.; Wrobel, L.C. An inverse geometry problem for the localisation of skin tumours by thermal analysis. Eng. Anal. Bound. Elem. 2007, 31, 803-811. [CrossRef]

30. Zhu, L. Fuzzy Inverse for Two-dimensional Steady Heat Transfer System and Application. Ph.D. Thesis, Chongqing University, Chongqing, China, 2011.

(C) 2019 by the authors. Licensee MDPI, Basel, Switzerland. This article is an open access article distributed under the terms and conditions of the Creative Commons Attribution (CC BY) license (http://creativecommons.org/licenses/by/4.0/). 